\title{
Spontaneous Pneumomediastinum in an Adolescent
}

\author{
Brig CM Adya, vsm*, Maj Vinay Maurya ${ }^{+}$Lt Col VD Charan\#, Brig AKS Bairaria**
}

MJAFI 2005; $61: 192-194$

Key Words : Pneumomediastinum

\section{Introduction}

$\mathrm{P}$ neumomediastinum (mediastinal emphysema) may be defined as the presence of free air or gas in the mediastinal structures [1]. It is an uncommon, but important condition found in healthy young adults and children presenting with chest pain and shortness of breath. Surgical emphysema may be widespread in such cases and may involve the neck, face, chest and arms. We report a case of 15-year-old boy who presented with acute onset shortness of breath and was found to have a spontaneous pneumomediastinum.

\section{Case Report}

A 15 year old, previously healthy male was admitted with a short history of one-day duration of moderate fever, dry irritant cough and sudden onset of shortness of breath. There was no history of chest pain, dysphagia, trauma or vomiting. On clinical examination he was ill looking, dyspnoeic, afebrile, pulse $-120 / \mathrm{min}$ regular, blood pressure-120/70 $\mathrm{mm} \mathrm{Hg}$, respiratory rate $-40 / \mathrm{min}$ thoraco-abdominal and there was no cyanosis. Subcutaneous emphysema was noted on the chest and neck. Respiratory examination revealed vesicular breath sounds and bilateral scattered crackles and medium pitched rhonchi. Cardiovascular auscultation was normal. There was no pulsus paradoxus and Hamman's sign was absent.

On investigations, Hb-13 gm\%, TLC-8900, DLC-P67 L27 M02 E 04. Liver function tests, blood urea, serum creatinine, blood sugar, and serum electrolytes were normal. ECG showed sinus tachycardia. Oxygen saturation was more than $95 \%$. Chest radiograph showed sharp line of lucency outlining the left border of the heart and air tracking along the fascial planes in the neck (Fig 1). CT chest showed extensive mediastinal emphysema with free air outlining the mediastinal structures (Fig 2). Extensive surgical emphysema was noted along the fascial planes in the neck, chest, axilla and back (Fig 3). Patchy areas of ground glass opacities were seen in both upper and left lower lobes along with thin walled dilated bronchi in both lower lobes (Fig 4). CT findings were suggestive of acute alveolitis with extensive mediastinal and surgical emphysema.
Patient was treated with antibiotics, oxygen inhalation and steroids for alveolitis and kept under observation. Recovery was uneventful with complete resolution of alveolitis and surgical emphysema in six days.

\section{Discussion}

Air in the human body is usually confined to the respiratory or the gastrointestinal tract but when the free air enters the mediastinum it is termed as mediastinal emphysema or pneumomediastinum [1]. Pneumomediastinum commonly results from alveolar rupture, in which case air tracks along interstitial and vascular supporting tissues till it reaches the mediastinum [2]. Rise in intrathoracic pressure during cough and straining is known to precipitate the condition as noted in common respiratory infections, asthma, whooping cough, alveolitis, bronchitis, foreign body choking, parturition and volutrauma during mechanical ventilation.

It can occur with blunt thoracic trauma, esophageal rupture during vomiting or esophagoscopy, therapeutic bronchoscopy, acute lung injury due to Mendelson's syndrome and mediastinitis by gas forming organisms. It has also been reported in cases of pulmonary tuberculosis, pneumocystic carinii pneumonia and ecstasy abuse $[3,4]$. No preexisting cause may be apparent in some cases.

Dyspnea and chest pain is the commonest presenting symptom. Chest pain is central and mimics myocardial infarction (MI) or pericarditis with exacerbation by movements and deep breathing and relief by leaning forward in sitting position. Dysphagia and dysphonia are also common. On examination, widespread surgical emphysema may be noted. Pulsus paradoxus may be felt and cardiac sounds may be muffled.

A characteristic, though not specific sign, noted in $50 \%$ cases is the Hamman's sign, a crackling or crunching sound heard at the sternal edge with patient

${ }^{*}$ Consultant (Medicine \& Rheumatology), INHS Asvini, Mumbai, ${ }^{+}$Graded Specialist (Radiology), 151 Base Hospital, C/o 99 APO, ${ }^{\#}$ Classified Specialist (Medicine \& Clinical Hematologist), Command Hospital (Southern Command) Pune-40, ${ }^{* *}$ DDMS, HQ 4 Corps (Med), C/o 99 APO

Received : 21.07.2003; Accepted : 24.01.2004 


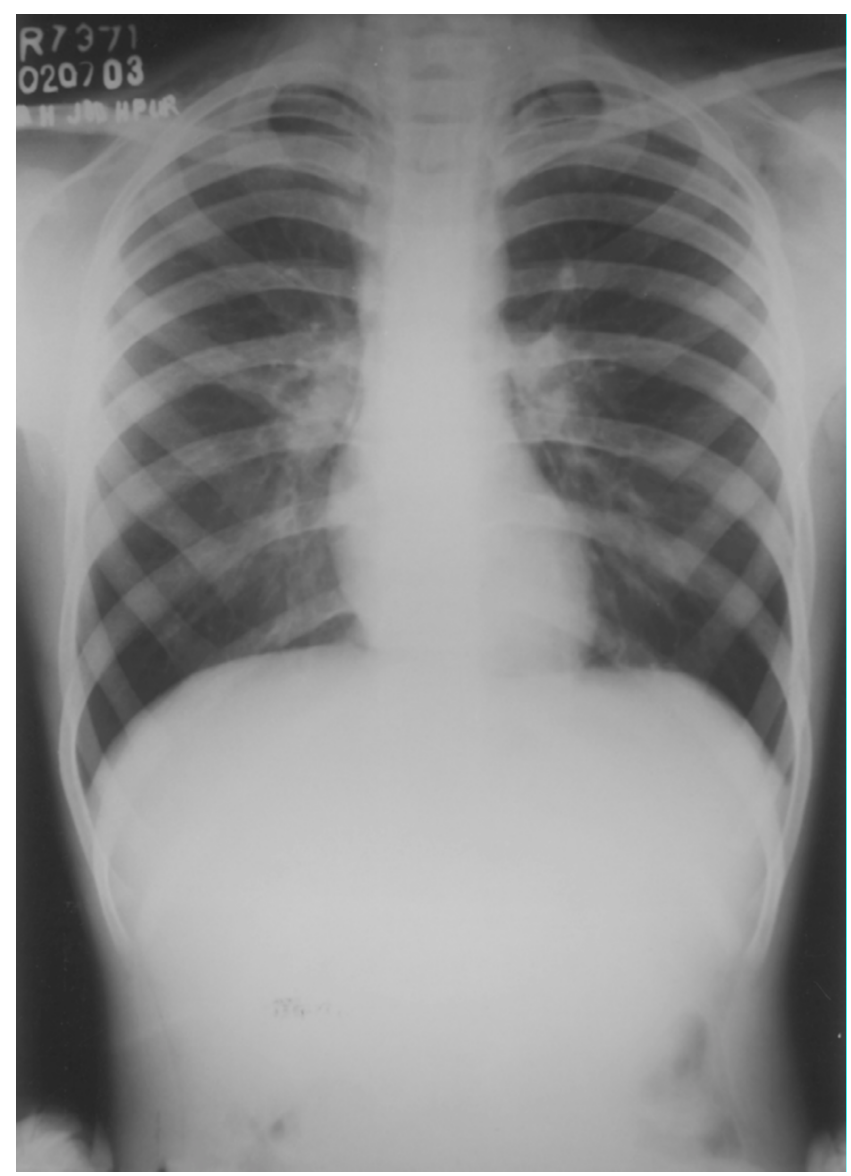

Fig. 1: Frontal radiograph chest shows sharp line of lucency outlining the left heart border and surgical emphysema in the neck

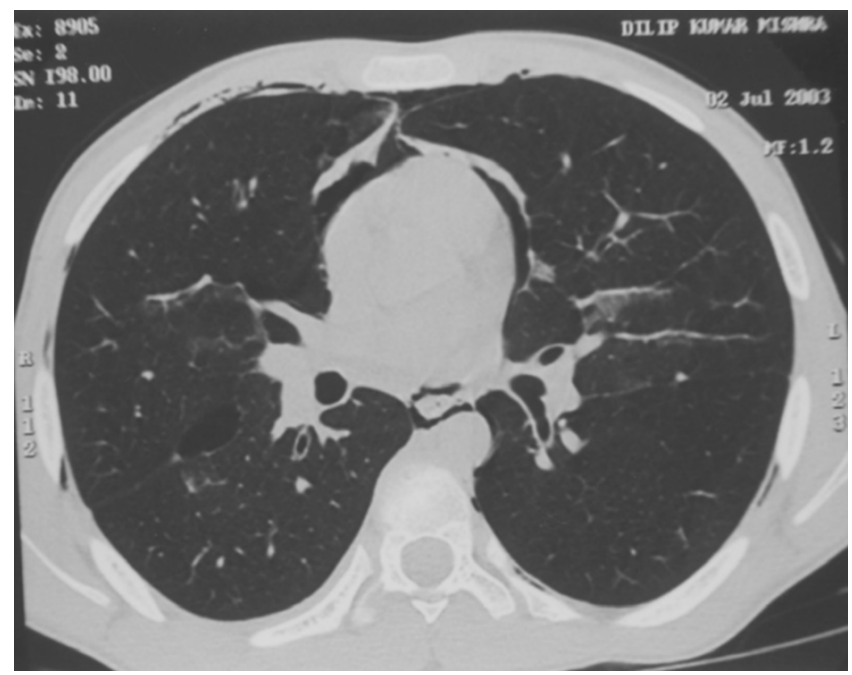

Fig. 2: CT chest demonstrates the air outlining inner surface of mediastinal pleura and the mediastinal structures

sitting forward or in the left lateral decubitus position [5].

ECG shows diffuse low voltage $\mathrm{ST}$ and $\mathrm{T}$ wave and loss of R-waves in the precordial leads in a standard 12-lead electrocardiogram, suggestive of an anterior wall MI [6]. Multifocal atrial tachycardia has been reported

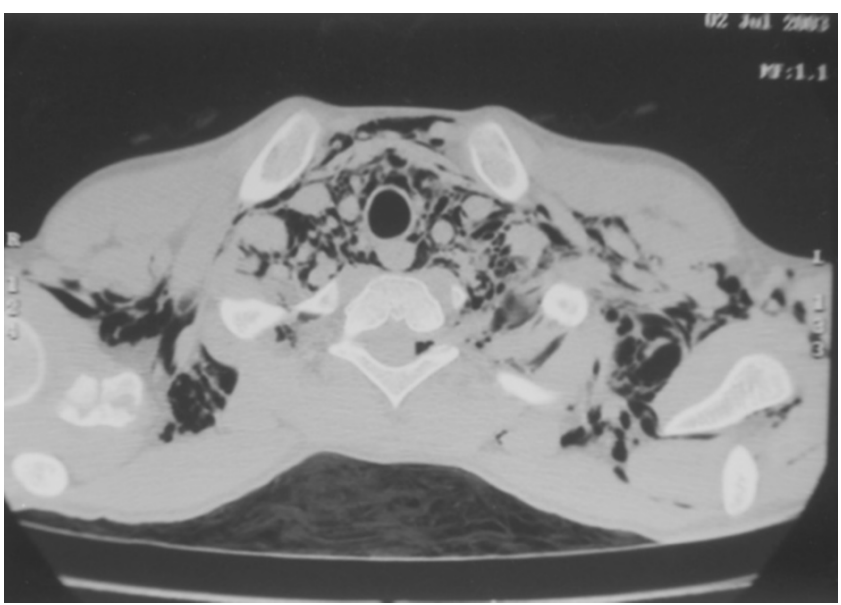

Fig. 3 : CT scan showing extensive surgical emphysema in the upper chest and axilla

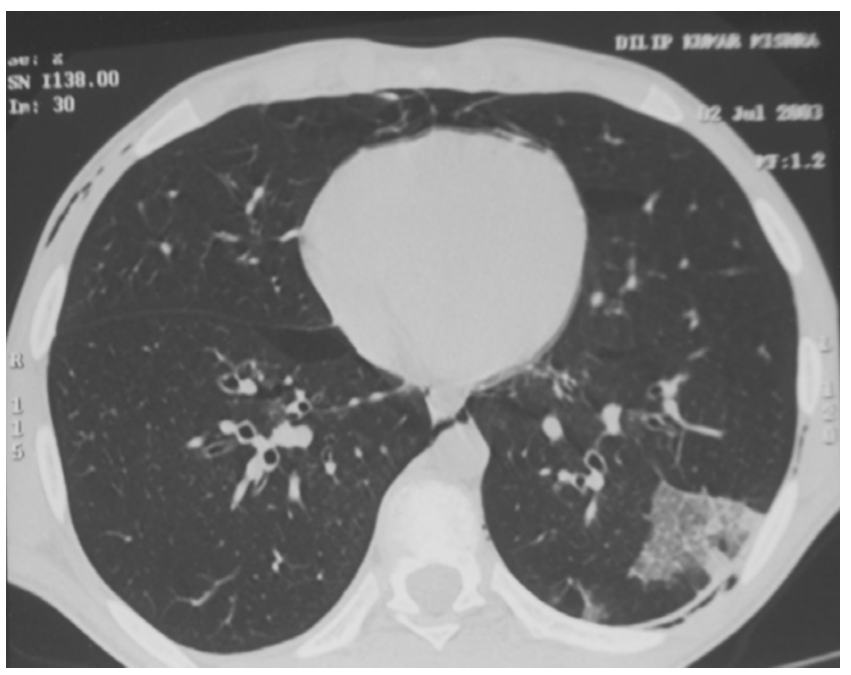

Fig. 4 : CT scan showing patchy area of ground glass opacity in the left lower lobe

in association with pneumomediastinum.

Radiographically pneumomediastinum is manifested by lucent streaks or bubbles of gas in the mediastinal soft tissues outlining mediastinal structures, elevate the mediastinal pleura and often extend into the neck or chest wall. Mediastinal air between the heart and the superior surface of the diaphragm results in "continuous diaphragm sign" on the frontal chest radiograph [7]. Nacleiros V sign refers to gas outlining the lateral margin of descending aorta and extending laterally between the parietal pleura and medial hemidiaphragm. The ring around the artery sign is formed by gas surrounding the extrapericardial portion of the right main pulmonary artery. In some cases pneumomediastinum can be confused with pneumothorax or pneumopericardium, but analysis of the distribution of the gas and accompanying signs will usually distinguish these conditions. Because gas in pneumomediastinum, unlike in pneumotharax and pneumopericardium, does not change in distribution with change in patient position, a contralateral decubitus view 
can be obtained to determine whether the gas shifts or not. CT demonstrate streaks of air in the mediastinum and, more importantly, other associated mediastinal, pleuroparenchymal and chest wall abnormalities [8].

Spontaneous pneumomediastinum seldom requires any treatment other than supportive care, reassurance, and analgesia [9]. Treatment or removal of precipitating cause is followed by gradual resolution of aberrant air. Administration of high concentration of oxygen may be beneficial in the rare circumstances in which diastolic filling of heart is impaired due to pneumomediastinum.

\section{References}

1. Kamath PG, Jadhav UM. Primary spontaneous pneumomediastinum. JAPI 2002;50:613-4.

2. Mocklin MT, Maclin CC. Malignant interstitial emphysema of the lungs and mediastinum as an important occult complication of many respiratory diseases and other condition: An interpretation of the clinical literature in the light of laboratory experiment. Medicine 1994;23:281-5.
3. Moss S, Carey PB, Hind CR. Pneumocystic carinii pneumonia presenting with pneumomediastinum. Postgraduate Medical J 1995;71:96-8.

4. Mazur S, Hitchcock T. Spontaneous pneumomediastinum, pneumothorax and ecstacy abuse. Emerg Med (Fremantle) 2001;13(1):121-3.

5. Bladt D, Bekaert I, Van Durme JP. Hamman's sign (pneumomediastinum-pneumopericardium). Acta Clin Belg 1981;36(1):5-11.

6. Zimmermann A, Rafii SE, Strom JA. Subcutaneous emphysema and pneumomediastinum: a cause of low-voltage electrocardiogram. Heart Dis 2001 Mar-Apr;3(2):85-6.

7. Levin B. The continuous diaphragm sign. Clin Radiol 1973;24:337-9.

8. Miele V, Andreoli C, De Cicco ML, et al. Spontaneous pneumomediastinum: radiologic and CT study. Minerva Chir 2000 Dec;55(12):869-72.

9. Bratton SL, O'Rourke PP. Spontaneous pneumomediastinum. J Emerg Med 1993 Sep-Oct;11(5):525-9. 